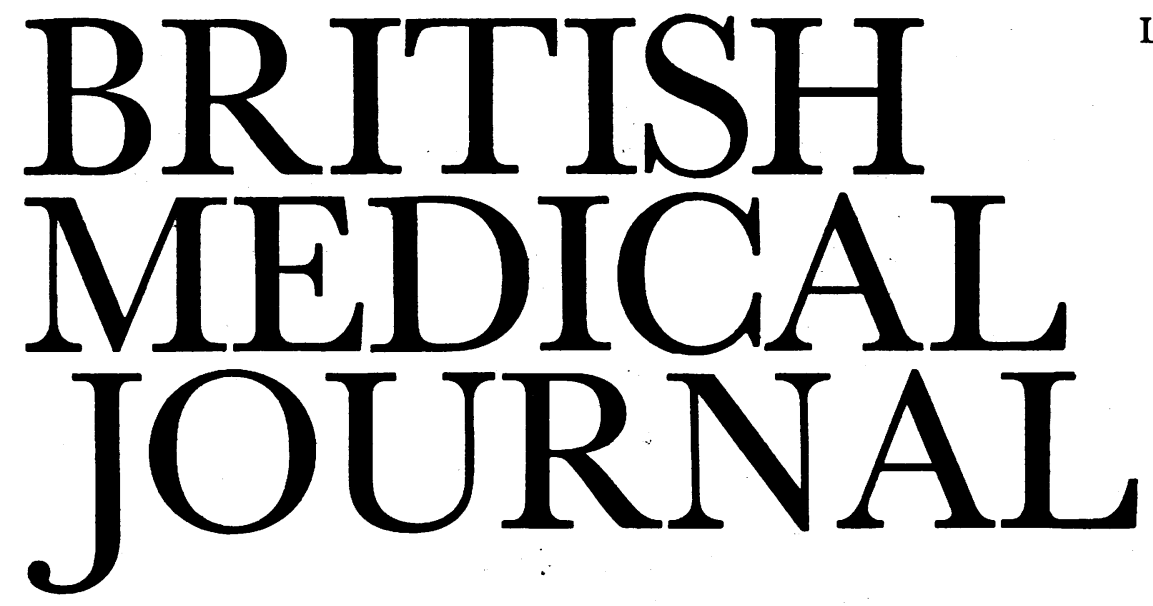

LONDON, SATURDAY 31 JANUARY 1987

\title{
Dangers in treating hyponatraemia
}

Serum sodium concentration is measured by hospital laboratories in most countries of the world, and yet the result is usually unhelpful. When the concentration is abnormal (usually reduced) the cause may be one of many conditions that impair the body's capacity to excrete water. A low serum sodium concentration is, in other words, usually the consequence of ill health, and treatment is best directed at the cause rather than its biochemical manifestation. Attempts at treating acute hyponatraemia can, however, have the most drastic consequences.

Occasionally chronic hyponatraemia is a predominant clinical problem-for instance, when inappropriate secretion of antidiuretic hormone by a tumour is responsible. Under these circumstances serum sodium concentration could be raised by water restriction or by giving potassium, which is predominantly retained within the cells and therefore promotes osmotic movement of water (but not sodium) into the cells. ${ }^{1}$ Both of these manoeuvres are of limited value, and the doctor may have to use drugs such as lithium or demeclocycline which enhance the renal excretion of water. ${ }^{2}$

The emergency treatment of severe hyponatraemia is much more contentious since there is as much potential for harm as for benefit. Water retention gives rise to dilution of extracellular fluid and therefore creates a short lived osmotic gradient between fluid inside and outside the cell. Water moves into the cell and causes cellular oedema. Mechanisms that regulate volume are probably then activated and lead to extrusion of intracellular cations and a corrective reduction in cell volume. ${ }^{3}$ At this stage the cell may suddenly be more vulnerable to further shrinkage if extracellular osmolality is suddenly raised. There is good clinical evidence that rapid changes in the hydration of brain cells can cause serious neurological problems including fits and coma. Temporary cellular overhydration, for instance, probably contributes to the dialysis disequilibrium syndrome when blood urea concentration is suddenly reduced by haemodialysis. ${ }^{4}$ In animals seizures produced by a rapid fall in serum sodium concentration are accompanied by an increase in the water content of the brain. ${ }^{5}$ Conversely a rapid rise in serum osmolality is associated with a rapid fall in cerebrospinal fluid pressure as the content of brain water falls: cerebral haemorrhage is a risk at this stage, particularly in children. ${ }^{6}$

Investigating rapid changes in serum sodium concentration is difficult, and not surprisingly, divergent advice is often given. Two recent reports underline this difficulty.
Arieff has reviewed his experience in treating 15 patients with severe hyponatraemia causing fits after elective surgery, none of whom had any relevant pre-existing medical condition. ${ }^{7}$ All were women, and giving fluids that contained glucose but were free of sodium seemed to be the most important factor. From operation to the first grand mal fit, which occurred an average of 49 hours later, patients were in a net positive fluid balance of $7 \cdot 5$ litres-sufficient to account for the observed fall in serum sodium concentration to 108 $\mathrm{mmol} / \mathrm{l}$. Normally this fluid could be excreted, but water excretion was probably impaired because surgery stimulated secretion of antidiuretic hormone. No patient recovered fully: four died, nine were left in a "persistent vegetative state" requiring care in an institution, and two had limb paralysis. They were treated with intravenous sodium chloride in concentrations ranging from isotonic to the very hypertonic ( $154 \mathrm{mmol} / \mathrm{l}$ up to $856 \mathrm{mmol} / \mathrm{l}$ ) and often with frusemide as well. Serum sodium concentration was thought to rise slowly in all patients (less than $0.7 \mathrm{mmol} / \mathrm{h}$ ), but the concentration fell more rapidly after operation in the patients who died.

Our limited understanding of the physiology of this condition would suggest that the rate of change of serum sodium concentration is important and that central pontine myelinosis may result from too rapid correction of hyponatraemia.$^{89}$ In another recent report Sterns $e t a l^{10}$ describe eight patients with hyponatraemia and neurological signs suggestive of this condition. Worsening of neurological function appeared to be associated with correcting serum sodium concentration at a rate over $12 \mathrm{mmol} / \mathrm{l}$ a day. A retrospective study of hospital records yielded 62 episodes of hyponatraemia over five years, and in 43 hyponatraemia had been corrected at this rapid rate. Five of these patients had neurological sequelae. Correction of hyponatraemia had been slower in the other 19 episodes, and none of the patients had suffered neurological sequelae.

Such analyses are fraught with the dangers of systematic bias. Patients treated with "rapid" or "slow" correction were unlikely to be comparable. A randomised prospective comparative trial may be set up, as Nairns suggests, ${ }^{11}$ but I doubt it. Many doctors will not participate in a study where there is a strong and widely held (but scientifically unproved) view that one of the treatments is harmful and produces severe permanent neurological damage or death.

The most obvious lesson from Arieff's paper is that large 
volumes of sodium free fluids should not be infused into patients whose diuretic capacity has been impaired by, for instance, an operation. Otherwise, without strong clinical evidence, there would seem sufficient physiological support for the view that rapid changes in serum sodium concentration (in either direction) are harmful. Thus the rate for correcting hyponatraemia suggested by Sterns et al seems reasonable. When hyponatraemia is not accompanied by important neurological dysfunction-as is usually the case, for instance, in patients treated with diuretics or with systemic illness-rapid parenteral correction is not justified as it may give rise to acute or permanent brain damage. Stopping the diuretics or treating the underlying illness is the most useful intervention.

Professor of Medicine,

J D SwAlES

University of Leicester,

Leicester Royal Infirmary,

Leicester LE2 7LX

1 Anonymous. Hyponatraemia. [Editorial.] Lancet 1978;i:642-4.

2 Forrest JN, Cox M, Hong L, Morrison G, Bia M, Singer I. Superiority of demaclocycline over lithium in the treatment of chronic syndrome of inappropriate secretion of antidiuretic hormone. N Engl f Med 1978;298:173-7.

3 Hoffman EK. Role of separate $\mathrm{K}^{+}$and $\mathrm{C}^{-}$channels and of $\mathrm{Na}^{+} / \mathrm{C}^{-}$-cotransport in volume regulation in Ehrlich cells. Fed Proc 1985;44:2513-9.

4 Kennedy AC, Linton AL, Renfrew S, Luke RG, Dinwoodie A. The pathogenesis and prevention of cerebral dysfunction during dialysis. Lancet 1964;i:790-3.

5 Hogan GR, Dodge PR, Gill SR, Masters S, Sotos JF. Pathogenesis of seizures occurring during restoration of plasma tonicity to normal in animals previously chronically hypernatramic. Pediatrics 1969;43:54-64.

6 Kravath RE, Aharon AS, Abal G, Finberg L. Clinically significant physiological danger from rapidly administered hypertonic solutions-acute osmol poisoning. Pediatrics 1970;46:267-75.

Arieff AI. Hyponatremia, convulsions, respiratory arrest and permanent brain damage after rieff AI. Hyponatremia, convulsions, respiratory arrest and perma
elective surgery in healthy women. NEngl f Med 1986;314:1529-35.

8 Kleinschmidt-DeMasters BK. Norenberg MD. Rapid correction.

of hyponatremia causes demyelination: relation to central pontine myelinosis. Science 1981;211:1068-70.

Laureno R. Rapid correction of hyponatremia: cause of pontine myelinosis? Am $\mathcal{J}$ Med 1981;71:846

10 Sterns RH, Riggs JE, Schochet SS. Osmotic demyelination syndrome following correction of hyponatremia. N Engl f Med 1986;394:1535-42.

11 Nairns RG. Therapy of hyponatremias. Does haste make worse? N Engl f Med 1986;314:1573-4.

\section{Restless legs syndrome}

The restless legs syndrome is characterised by an unpleasant creeping sensation deep in the legs. It usually comes on in the evenings and when the patient is at rest, and walking will generally bring relief. ${ }^{1}$ Patients may find these symptoms difficult to describe-sensations are felt deep in the muscles or bones as if they are "crawling with ants" or full of "writhing worms." These bizarre descriptions may sound almost delusional, but this is not primarily a psychiatric condition. Symptoms are usually bilateral but can occur in one leg. Most commonly they are experienced in the calf but sometimes in the thigh and foot; rarely they spread to the arms. The compulsion to move once these sensations begin is the most important diagnostic feature as usually no abnormalities are found on examination. The restless legs syndrome is an important cause of insomnia and may be associated with periodic movements in sleep also known as nocturnal myoclonus. ${ }^{2}$

Willis described the syndrome in $1685,{ }^{3}$ but it was poorly recognised until Ekbom's series of papers-and his name is rightly given to the condition. ${ }^{1}$ (Other names, such as asthenia crurum, anxietas tibiarum, and irritable legs, have disappeared.) The restless legs syndrome affects up to $5 \%$ of the population at some time. Men and women are equally affected at any age, though it is more common in the elderly. ${ }^{4}$ Usually the condition is chronic, but it may occur first during pregnancy and then remit. Familial occurrence is said te occur but is poorly documented, though families witk apparent autosomal dominance are known. ${ }^{2}$ Poliomyelitis? avitaminosis, diabetes, smoking, Parkinson's disease, andlengthy exposure to cold have all been associated with the restless legs syndrome, but the only certain associations are with pregnancy, iron deficiency anaemia, and uraemia. I I patients with uraemia the syndrome may herald uraemign neuropathy. ${ }^{5}$ A third of patients with rheumatoid arthritis. have the restless legs syndrome, which may be related to irow deficiency. ${ }^{6}$

The origin of the sensations is unknown. There are nळ pathological abnormalities of muscle or nerve. ${ }^{7}$ Ekbone thought a vascular pathogenesis likely and recorded succes $\stackrel{\oplus}{.}$ with vasodilator drugs such as tolazoline and nitroglycerin. Others have suspected a more central origin-perhaps in the spinal cord or basal ganglia. The resemblance of the restless. legs syndrome to akathisia after neuroleptic drugs hass suggested a neurochemical basis such as an imbalance between serotonergic and dopaminergic pathways, ${ }^{8}$ bue there are important differences between the two conditionsto akathisia is motor restlessness arising primarily from psychological desire, whereas the desire to move in the restless legs syndrome arises directly from the leg dis comfort. This difference should distinguish the two.

Because the pathophysiology of the restless legs syndrome has remained obscure treatment has been empirical ando many claims have been made. Unfortunately few properly $\overrightarrow{\mathrm{L}}$ controlled trials have been carried out, and it is rare to see an criteria given for diagnosis. Recently Gibbs and Lee have suggested criteria for diagnosis that should help in future trials. ${ }^{9}$ Many patients will not need drug treatment; re ${ }_{\infty}^{\circ}$ assurance on the benign nature of their condition may weli suffice. Others will manage by taking a walk or by wriggling their feet to and fro when symptoms arise. Doctors should not belittle symptoms. A minority of patients find their live $\overline{8}$ ruined: the need to keep moving may lead them to avoi social engagements. Insomnia too can be a great problem? $\frac{2}{2}$ and these patients need sympathetic handling.

It is probably best to explain the limitations of drug treatment early on as disillusionment with the doctor because of repeated drug failures is common. Many drugs have been used in the restless legs syndrome-vasodilators, ${ }^{2}$ clonidine, carbamazepine, ${ }^{11}$ triazolam, clonazepam, ${ }^{12}$ methysergide tryptophan, ${ }^{13}$ iron, folate, ${ }^{3}$ propranolol, chlorpromazine, amytriptyline, and procaine infusions. ${ }^{14}$ Only clonazepang and carbamazepine have shown any benefit over placebo. ${ }^{10} 15$

Recently Von Scheele has advocated using levodopa. ${ }^{1 \delta}$ Akpinar first used levodopa and benserazide (Madopar) in five patients in an open trial and thought it helped. ${ }^{8}$ Vor Scheele's results were more substantial -17 out of $2 \Phi$ patients preferred levodopa (plus benserazide) to lactose in a double blind trial and all reported complete relief. This is anv astonishing result, and I hope these results can be confirmed by others. There were problems with the trial: no diagnostic criteria were given, and the follow up was only one to twक् weeks. A long term study should follow.

What practical advice can be given to the many doctors? who are faced with this disorder? We should be aware of the common occurrence of the restless legs syndrome and make the diagnosis on the basis of a characteristic history and the absence of physical signs. Iron deficiency anaemia and uraemia should be looked for. Most patients will not neeф treatment, but for those who do I suggest a trial of the following (the order is dictated more by side effects tharf proved efficacy): levodopa (and benserazide or carbidopa); 